\title{
Optical observations of a newly identified compact galaxy group near the Zone of Avoidance ${ }^{\star}$
}

\author{
S. Temporin ${ }^{1}$ and S. Ciroi ${ }^{2}$ \\ 1 Institut für Astrophysik, Universität Innsbruck, Technikerstraße 25, 6020 Innsbruck, Austria \\ 2 Dipartimento di Astronomia dell' Universitá di Padova, Vicolo dell’Osservatorio 2, 35122 Padova, Italy \\ e-mail: ciroi@pd.astro.it
}

Received 11 September 2002 / Accepted 22 October 2002

\begin{abstract}
We have identified a new group of galaxies, CG J0247+44.9, at low galactic latitude $\left(l=143^{\circ} .64, b=-13^{\circ} .29\right)$, which satisfies Hickson's criteria (Hickson 1997 for Compact Groups (CGs)). Our group consists of six members, two of which are in close interaction (IRAS $02443+4437$ ).

We present here optical photometry $(B V R I)$ and low resolution spectroscopy of the individual galaxies and investigate the global properties of the group. Our morphological analysis reveals that two out of the six objects are lenticular galaxies. The others are spirals showing emission lines in their spectra through which we could classify them as a starburst galaxy (the spiral member of the IRAS $02443+4437$ close pair), a Seyfert 2, a LINER and a weak HII galaxy. Since the S0/Sa is the prevailing morphology for the galaxies of this group, which is also characterized by a short crossing time and a relatively high velocity dispersion, we suggest that CG J0247+44.9 is a dynamically old compact group.
\end{abstract}

Key words. galaxies: clusters: individual: CG J0247+44.9 - galaxies: photometry - galaxies: interactions

\section{Introduction}

As it is well known, our view of the extragalactic space at low Galactic latitudes is obscured by a patchy layer of dust, whose inner part $\left(|b| \lesssim 10^{\circ}\right)$ is called Zone of Avoidance (ZoA). However, numerous multi-wavelength surveys carried out by different research groups (e.g. Nakanishi et al. 1997; Kraan-Korteweg \& Lahav 2000; Weinberger et al. 2000, and references therein) in the last decades led to the detection of tens of thousand of galaxies in this band of the Milky Way. Such a huge galaxy database has a high potential both for extragalactic studies and for studies of our own Galaxy. In particular we started a project (S. Temporin et al., in preparation) devoted to investigate the dust distribution in the Milky Way in dependence on Galactic longitude and latitude. This purpose will be achieved by evaluating the total foreground Galactic extinction along many lines-of-sight to galaxies in the ZoA and its vicinity through $B V R I C C D$-photometry. Indeed, depending on their morphological type, galaxies have well-defined intrinsic total and effective optical colors (Buta \& Williams 1995), which can be used to determine the color excess and then the visual absorption $A_{\mathrm{V}}$. In order to largely avoid the fine-scale structure of Galactic dust (clumpiness) we selected close pairs

Send offprint requests to: $\mathrm{S}$. Temporin,

e-mail: giovanna.temporin@uibk.ac.at

* Based on data obtained at Calar Alto (Spain) and Asiago (Italy) observatories. and/or multiple systems of galaxies to reduce the errors in the determination of Galactic extinction along a specific line-ofsight.

As an aside we have performed a spectroscopic followup of these selected systems, whose physical properties are still widely unknown, in order to establish, through the determination of their radial velocities, which of them are physically bound and not only chance projections on the plane of the sky. We have identified in this way a new compact galaxy group at low galactic latitude $\left(l=143^{\circ} .64, b=-13^{\circ} .29\right)$, to which we refer here as CG J0247+44.9, containing six members. Two of them form a close interacting pair, already known as IRAS $02443+4437$, other three galaxies are cataloged in the 2MASS survey (Huchra et al. 2000) and the last one is uncataloged. We present here the analysis of photometric (Sect. 2) and spectroscopic data (Sect. 3) of this group. We discuss the individual properties of its members in Sect. 4 and the global properties of the group in Sect. 5. The main results are summarized in Sect. 6.

\section{BVRI photometry}

Data in the broad-band BVRI Johnson-Cousins system were obtained under photometric conditions at the $1.23 \mathrm{~m}$ telescope of the Calar Alto Observatory (Spain) in January 1999. The images covered a field of view of $\sim 8^{\prime} .5 \times 8^{\prime} .5$ with a spatial scale 
Table 1. Observation logs.

\begin{tabular}{llllllll}
\hline \hline Telescope & Instrument & Filter/Grism & Date & $\begin{array}{l}T_{\text {exp }} \\
(\mathrm{s})\end{array}$ & $\begin{array}{l}\text { Seeing } \\
\left({ }^{\prime \prime}\right)\end{array}$ & $\begin{array}{l}\text { Scale } \\
(" \prime / p x)\end{array}$ & $\begin{array}{l}\text { Slit } \\
\left({ }^{\prime \prime}\right.\end{array}$ \\
\hline Calar Alto 1.23 m & CCD-camera & Johnson $B$ & $1999-01-06$ & 2100 & 1.4 & 0.5 & $\ldots$ \\
Calar Alto 1.23 m & CCD-camera & Johnson $V$ & $1999-01-06$ & 1200 & 1.1 & 0.5 & $\ldots$ \\
Calar Alto 1.23 m & CCD-camera & Cousins $R$ & $1999-01-06$ & 900 & 1.0 & 0.5 & $\ldots$ \\
Calar Alto 1.23 m & CCD-camera & Cousins $I$ & $1999-01-06$ & 300 & 1.1 & 0.5 & $\ldots$ \\
Asiago 1.82 m & AFOSC & $\# 4$ & $2001-09-13$ & 1800 & 1.2 & 0.47 & 1.26 \\
Asiago 1.82 m & AFOSC & $\# 4$ & $2001-11-25$ & 1800 & 2.2 & 0.47 & 2.10 \\
Asiago 1.82 m & AFOSC & $\# 4$ & $2001-11-26$ & $2 \times 1800$ & 2.2 & 0.47 & 2.10 \\
\hline
\end{tabular}

of 0.5 arcsec pixel $^{-1}$. Observational details are summarized in Table 1.

The images were reduced with $\mathrm{IRAF}^{1}$ following the standard steps of bias subtraction, flat fielding and cosmic rays removal. An additional defringing procedure was applied to the $I$ band image. A Landolt (1992) standard field was observed three times during the night for photometric calibration purposes. The calibration constants (see Table 2) were determined by means of the IRAF package PHOTCAL through a uniformly weighted fit of the following transformation equations:

$$
\begin{aligned}
& B=b+B_{0}-k_{B} X_{B}+C_{B}(B-V) \\
& V=v+V_{0}-k_{V} X_{V}+C_{V}(B-V) \\
& R=r+R_{0}-k_{R} X_{R}+C_{R}(V-R) \\
& I=i+I_{0}-k_{I} X_{I}+C_{I}(R-I)
\end{aligned}
$$

where $B, V, R$, and $I$ are calibrated magnitudes, $b, v, r$, and $i$ are sky-subtracted instrumental magnitudes normalized to $1 \mathrm{~s}$ exposure and $X_{B}, X_{V}, X_{R}$, and $X_{I}$ are the airmasses at the time of the observations.

We have identified seven galaxies in the field, two of which form an interactive pair known as IRAS 02443+4437 (Fig. 1). We have studied the morphology of each galaxy and calculated the total magnitudes in the four bands by means of GIM2D (Simard 1998; Simard et al. 2001), an IRAF package written to perform an automated bulge-disk decomposition of the surface brightness profile. GIM2D is well suited for low signal-to-noise images of distant galaxies since it has the considerable advantage of including a PSF deconvolution in the bi-dimensional fit. The effects of the distance in dimming the outer parts of galaxies' disks can be considered comparable to the effects produced by heavy Galactic foreground extinction in nearby galaxies. Therefore the suitability of the program to the bulge-disk decomposition of distant galaxies makes it also particularly appropriate in the case of low-Galactic-latitude galaxies - like those studied in this work - which might be heavily affected by foreground Galactic extinction.

${ }^{1}$ IRAF is distributed by the National Optical Astronomy Observatories, which are operated by the Association of Universities for Research in Astronomy, Inc., under cooperative agreement with the National Science Foundation.
Table 2. Photometric calibration constants ${ }^{\mathrm{a}}$.

\begin{tabular}{llll}
\hline \hline Band & $M_{0}$ & $k_{\mathrm{M}}$ & $C_{\mathrm{M}}$ \\
\hline$B$ & $22.193 \pm 0.054$ & $0.227 \pm 0.041$ & $0.110 \pm 0.009$ \\
$V$ & $22.624 \pm 0.041$ & $0.138 \pm 0.030$ & $-0.028 \pm 0.007$ \\
$R$ & $23.102 \pm 0.036$ & $0.086 \pm 0.026$ & $0.064 \pm 0.009$ \\
$I$ & $22.526 \pm 0.162$ & $0.071 \pm 0.117$ & $0.274 \pm 0.061$ \\
\hline
\end{tabular}

a With $M_{0}, k_{\mathrm{M}}$, and $C_{\mathrm{M}}$ we indicate the zero point, the extinction coefficient and color term, respectively, in each of the four photometric bands.

The program gives as output, the values of the best-fit parameters and asymmetry indices, besides an image of the galaxy model and a residual image obtained by subtracting the model from the original one. This fitting method has already been successfully applied by Tran et al. (2001) to a sample of galaxies belonging to poor galaxy groups and spanning a wide range in morphological type. They found that the ratio between the bulge and the total luminosity $(B / T)$ can be used on average as a robust morphology indicator to discriminate between early-type bulge-dominated $(B / T>0.4)$ and late-type diskdominated $(B / T<0.4)$ galaxies.

The PSF images in the four photometric bands to be used during the galaxy fitting procedure were obtained with the DAOPHOT (Stetson \& Harris 1988) package inside IRAF. The isophotal area of the galaxies was defined by means of the galaxy photometry package SExtractor V2.2.2 (Bertin \& Arnouts 1996). The fit uses an exponential law for the disk, while a classical de Vaucouleurs $r^{1 / 4}$-law (de Vaucouleurs 1948) or a Sérsic profile (Sérsic 1968) can be chosen for the bulge. In the last case, the $n$ index of the Sérsic law is one of the fitting parameters. We attempted fits of the seven galaxies with a Sérsic law plus exponential disk. When the $n$ index was equal or very close to 4, we applied a de Vaucouleurs law for the bulge. In Table 3 we list the best-fit parameters from GIM2D: bulge to total light ratio $(B / T)$, bulge effective radius $\left(R_{\mathrm{e}}\right)$, ellipticity $(e)$, disk scale length $\left(R_{\mathrm{d}}\right)$, inclination $(i)$, Sérsic law index $(n)$, half-light radius $\left(R_{\text {half }}\right)$, asymmetry index $\left(R_{\mathrm{A}}\right)$, together with the reduced $\chi^{2}$. We show in Fig. 2 the $B V R I$ 


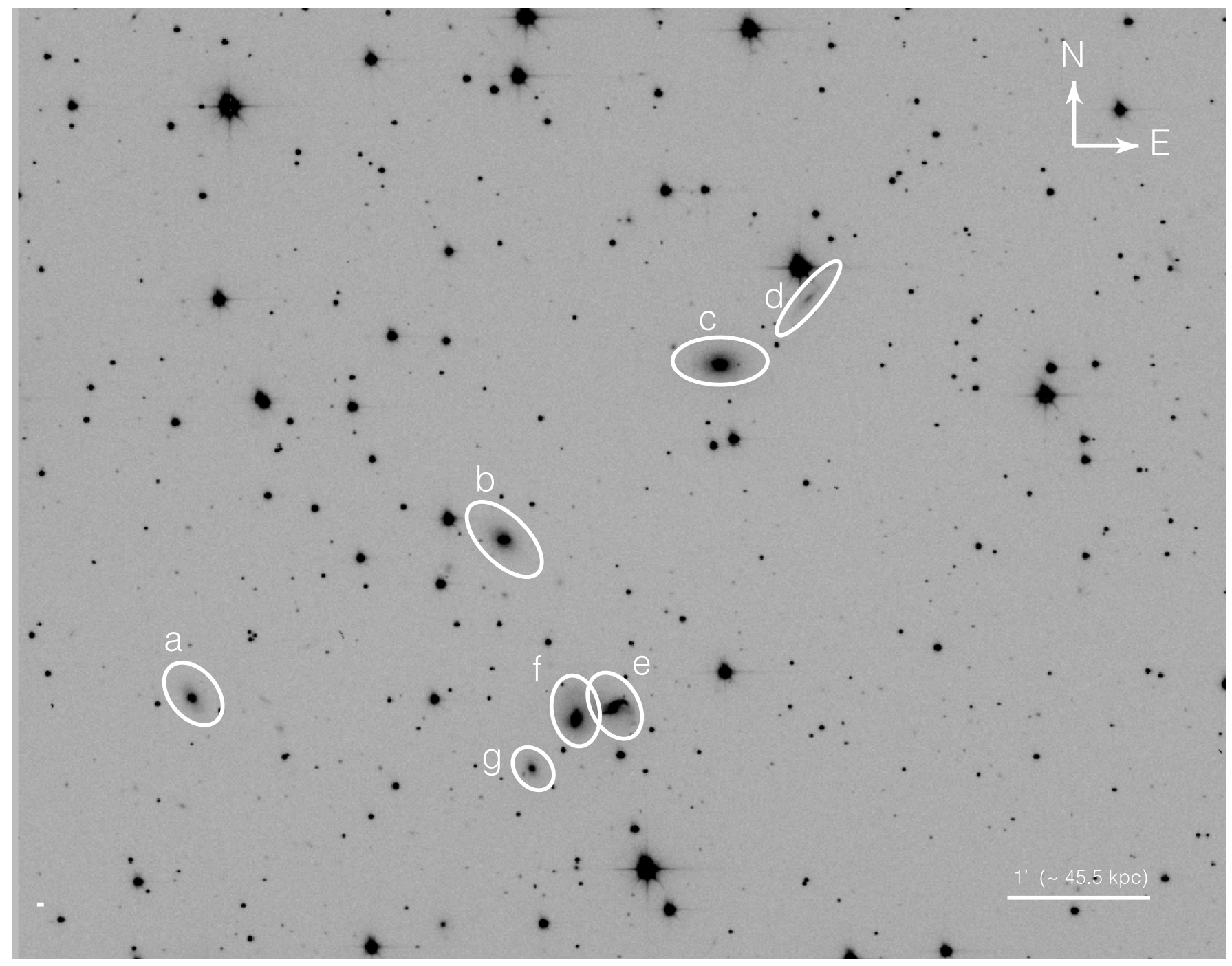

Fig. 1. $R$-band exposure of the galaxy system around the position $\alpha=02^{\mathrm{h}} 47^{\mathrm{m}} 36^{\mathrm{s}} \cdot 3, \delta=44^{\circ} 51^{\prime} 39^{\prime \prime}(\mathrm{J} 2000)$. The galaxies identified in the field of view are marked with ellipses and labeled with small-case letters. The minimum circle containing all geometrical centers of the galaxies is drawn, as well. Objects "e" and "f" are the components of the interactive pair IRAS 02443+4437.

thumbnails of each galaxy and in Fig. 3 the corresponding residuals after subtraction of the best-fit galaxy models.

The two parameters $B / T$ and $n$ were used for the morphological classification of the galaxies (see Sect. 4). Our $B / T$ values in the $B$-band were compared with those plotted as a function of the morphological type $T$ by Simien $\&$ de Vaucouleurs (1986). Additionally we took into account the growing evidence that late-type spirals host non-classical bulges, better reproduced by an exponential law $(n=1)$ (Andredakis \& Sanders 1994; Andredakis 1998; Carollo et al. 2001).

The inclination correction terms $A_{B_{i}}, E(B-V)_{i}$ and the selective extinction $R_{V}$ have been calculated as a function of the morphological type $T$ and of the $B$-band disk inclination $i$ (instead of the ratio $R_{25}$ between the major and minor diameters at the $\mu_{B}=25 \mathrm{mag} \operatorname{arcsec}^{-2}$ isophote) following the instructions given in the introduction to the Third Reference Catalogue of Bright Galaxies (de Vaucouleurs et al. 1991, RC3). The inclination corrections in the other photometric bands were derived by application of the Cardelli et al. (1989, CCM) extinction law. The inclination corrected total and effective colors $B-V$, $V-R$, and $V-I$ of three galaxies, selected for being sufficiently separated one another and not too much inclined, were used to estimate the total foreground Galactic extinction. For this purpose the three colors available for each galaxy were compared with the mean values for the relevant morphological type published by Buta \& Williams (1995), and the corresponding color excesses $E(B-V)$ were calculated and averaged. Finally the weighted mean of the values obtained for these three objects, $E(B-V)=0.29 \pm 0.06\left(A_{B}=1.19\right.$ mag, assuming a selective extinction $\left.R_{V}=3.1\right)$ was used as a measure of the Galactic extinction in the direction of the galaxy group. We stress that this value is significantly different from the ones available from $\mathrm{NED}^{2}, A_{B}=0.610$ and $A_{B}=0.642 \mathrm{mag}$, based on the maps of Schlegel et al. (1998) and Burstein \& Heiles (1982), respectively. This discrepancy could be due to smallscale fluctuations in the foreground extinction as a consequence of the patchy dust distribution at low Galactic latitudes. Such

${ }^{2}$ NASA extragalactic database. 
Table 3. GIM2D best-fit parameters.

\begin{tabular}{|c|c|c|c|c|c|c|c|c|c|}
\hline Band & $B / T$ & $R_{\mathrm{e}}\left({ }^{\prime \prime}\right)$ & $e$ & $R_{\mathrm{d}}\left({ }^{\prime \prime}\right)$ & $i\left(^{\circ}\right)$ & $n$ & $\chi^{2}$ & $R_{\text {half }}\left({ }^{\prime \prime}\right)$ & $R_{\mathrm{A}}$ \\
\hline \multicolumn{10}{|c|}{ CG J0247+449a } \\
\hline$B$ & 0.31 & 0.62 & 0.21 & 2.76 & 54 & 0.91 & 1.01 & 2.86 & 0.02 \\
\hline$V$ & 0.36 & 0.67 & 0.20 & 3.19 & 56 & 1.15 & 1.02 & 2.81 & 0.01 \\
\hline$R$ & 0.38 & 0.57 & 0.21 & 3.19 & 57 & 1.31 & 1.07 & 2.65 & 0.01 \\
\hline$I$ & 0.45 & 0.63 & 0.22 & 3.87 & 58 & 2.33 & 1.00 & 2.70 & 0.00 \\
\hline \multicolumn{10}{|c|}{ CG J0247+449b } \\
\hline$B$ & 0.59 & 1.08 & 0.20 & 4.06 & 56 & 4 & 1.05 & 2.74 & 0.02 \\
\hline$V$ & 0.57 & 0.97 & 0.28 & 3.82 & 52 & 4 & 1.04 & 2.61 & 0.01 \\
\hline$R$ & 0.59 & 0.95 & 0.22 & 4.19 & 53 & 4 & 1.11 & 2.51 & 0.02 \\
\hline$I$ & 0.55 & 0.80 & 0.26 & 4.05 & 48 & 4 & 1.03 & 2.57 & 0.01 \\
\hline \multicolumn{10}{|c|}{ CG J0247+449c } \\
\hline$B$ & 0.33 & 1.22 & 0.39 & 4.86 & 45 & 4 & 1.00 & 5.57 & 0.00 \\
\hline$V$ & 0.42 & 1.65 & 0.33 & 5.12 & 46 & 4 & 1.05 & 5.33 & 0.01 \\
\hline$R$ & 0.40 & 1.40 & 0.32 & 5.67 & 46 & 4 & 1.09 & 5.69 & 0.01 \\
\hline$I$ & 0.39 & 1.05 & 0.32 & 4.77 & 48 & 4 & 1.00 & 4.78 & 0.01 \\
\hline \multicolumn{10}{|c|}{ CG J0247+449d } \\
\hline$B$ & 0.22 & 3.99 & 0.69 & 9.29 & 76 & 1.64 & 1.07 & 12.44 & 0.10 \\
\hline$V$ & 0.84 & 14.91 & 0.70 & 34.54 & 85 & 2.48 & 1.22 & 18.63 & 0.07 \\
\hline$R$ & 0.60 & 8.88 & 0.70 & 15.29 & 73 & 2.01 & 1.13 & 14.09 & 0.07 \\
\hline$I$ & 0.94 & 13.25 & 0.41 & 2.33 & 82 & 1.64 & 1.07 & 12.31 & 0.08 \\
\hline \multicolumn{10}{|c|}{ CG J0247+449e } \\
\hline$B$ & 0.32 & 2.87 & 0.64 & 6.06 & 55 & 0.38 & 1.60 & 6.24 & 0.17 \\
\hline$V$ & 0.42 & 3.38 & 0.65 & 4.29 & 55 & 0.48 & 2.90 & 4.86 & 0.17 \\
\hline$R$ & 0.35 & 2.93 & 0.69 & 4.67 & 55 & 0.41 & 3.00 & 5.06 & 0.16 \\
\hline$I$ & 0.39 & 2.86 & 0.67 & 6.30 & 60 & 0.73 & 1.40 & 5.95 & 0.09 \\
\hline \multicolumn{10}{|c|}{ CG J0247+449f } \\
\hline$B$ & 0.77 & 3.17 & 0.38 & 4.80 & 60 & 4 & 1.17 & 4.27 & 0.02 \\
\hline$V$ & 0.65 & 2.86 & 0.39 & 5.68 & 43 & 4 & 1.16 & 4.98 & 0.04 \\
\hline$R$ & 0.73 & 3.04 & 0.42 & 5.92 & 54 & 4 & 1.44 & 4.61 & 0.04 \\
\hline$I$ & 0.67 & 2.53 & 0.46 & 4.97 & 45 & 4 & 1.17 & 4.21 & 0.02 \\
\hline \multicolumn{10}{|c|}{ CG J0247+449g } \\
\hline$B$ & 0.66 & 2.60 & 0.34 & 3.00 & 69 & 4 & 1.00 & 3.57 & 0.01 \\
\hline$V$ & 0.72 & 3.27 & 0.41 & 5.64 & 59 & 4 & 0.99 & 4.86 & 0.01 \\
\hline$R$ & 0.64 & 2.12 & 0.40 & 3.23 & 25 & 4 & 1.00 & 3.34 & 0.01 \\
\hline$I$ & 0.53 & 1.51 & 0.51 & 3.42 & 38 & 4 & 1.08 & 3.34 & 0.02 \\
\hline
\end{tabular}

fluctuations cannot be detected by interpolating data from large scale maps like those cited above. In Table 4 we list observed magnitudes and colors of the seven galaxies in the field and the corresponding values (in bold) corrected for inclination and Galactic extinction.

\section{Spectroscopy}

Long-slit spectra of the individual galaxies were obtained during two different runs in September and November 2001 at the $1.82 \mathrm{~m}$ telescope of the Asiago Observatory (Italy) equipped with AFOSC, which has a spatial scale of $0.47 \operatorname{arcsec}_{\text {pixel }}{ }^{-1}$ over a $1 \mathrm{k} \times 1 \mathrm{k} C \mathrm{CD}$. The grism $\mathrm{n} .4$, chosen for its large spectral coverage, gave a dispersion of $4.2 \AA$ pixel $^{-1}$ in the effective range of 4200-7700 $\AA$. A slit width of 1.26 and 2.1 arcsec, selected according to the seeing conditions, produced a spectral resolution of $\sim 13 \AA$ and $24 \AA$ in the two runs, respectively. Typical exposure times were of $1800 \mathrm{~s}$ each. See Table 1 for details about the observations.

Spectra were reduced with IRAF following the usual steps of bias subtraction, flat-field correction, cosmic-rays removal, wavelength calibration by means of Helium-Argon or Thorium comparison lamps and night-sky subtraction. Finally they were flux calibrated through the observation of the 


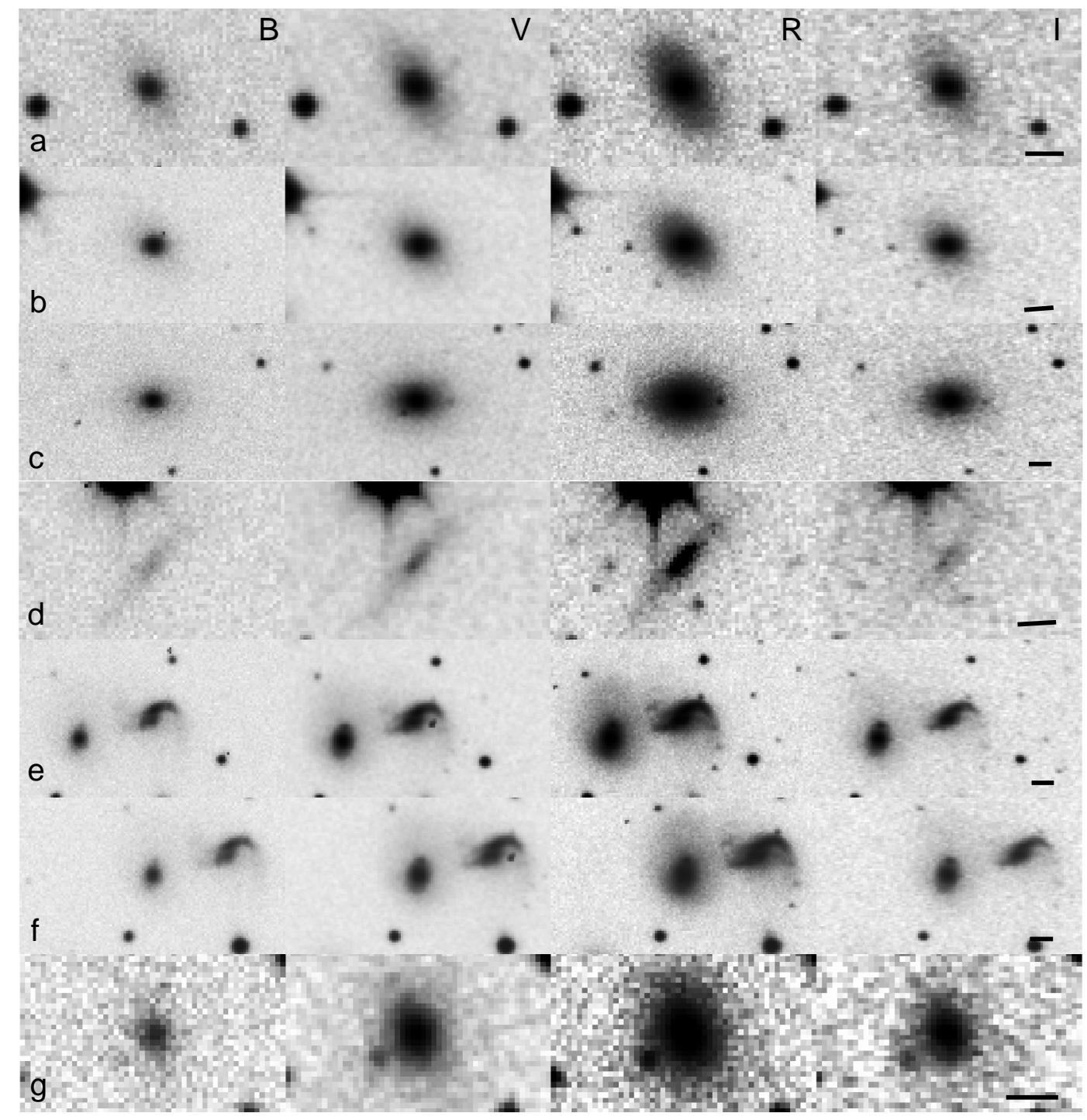

Fig. 2. Thumbnails showing the $B V R I$-band images of the seven galaxies identified in Fig. 1. The short bars on the bottom-right corner of the $I$ images of each galaxy are 5 arcsec in size.

spectrophotometric standard stars BD+284211 and G191-b2b. The 1D spectra obtained adding the total flux of each object with the task APALL were corrected for Galactic extinction using the value $E(B-V)=0.29$ derived above and applying the CCM extinction law.

Radial velocities were calculated by measuring the position of the emission-lines, where detectable, or by using the cross-correlation technique Tonry \& Davis (1979) when only the absorption lines were present. Six out of seven galaxies show comparable redshifts (Table 5), suggesting that they are members of a group; their spectra are shown in Fig. 4. Only in the case of galaxy "g" (2MASXi J0247387+445008) it was impossible to apply the above methods due to the low $S / N$ ratio of the spectrum. Nevertheless we could recognize some absorption lines by comparing the spectrum with an early-type galaxy template ${ }^{3}$ Kinney et al. (1996) and we estimated a redshift of $\sim 0.17$. This estimate is in agreement with the observed color

\footnotetext{
${ }^{3}$ Galaxy template spectra by Kinney et al. (1996) are available from ftp://ftp.stsci . edu/cdbs/cdbs2/grid/kc96/
}

$B-R=2.13$, considerably redder than typical colors for the same morphological type at low redshift (see e.g. Fig. 2 of Liu 1999). Therefore object " $\mathrm{g}$ " appears to be a background galaxy and not a member of the group.

\section{Notes on individual members}

CG J0247+449a. The morphological analysis in all four photometric bands indicates a regular galaxy without asymmetric structures. It is well fitted by an exponential bulge $(n \sim 1)$ and the $B / T$ ratio is $\sim 0.37$. No residuals are visible after model subtraction (see Fig. 3). For this object (also known as 2MASXi J0247252+445039) we suggest a morphological type $\mathrm{Sa} / \mathrm{Sab}$. The optical spectrum shows weak continuum and emission lines (Fig. 4). [O III]5007 is the brightest emission line, while Balmer hydrogen lines are partly absorbed by the underlying stellar continuum. After the subtraction of this stellar contribution by means of a template galaxy spectrum, following the prescriptions by Ho et al. (1993), 


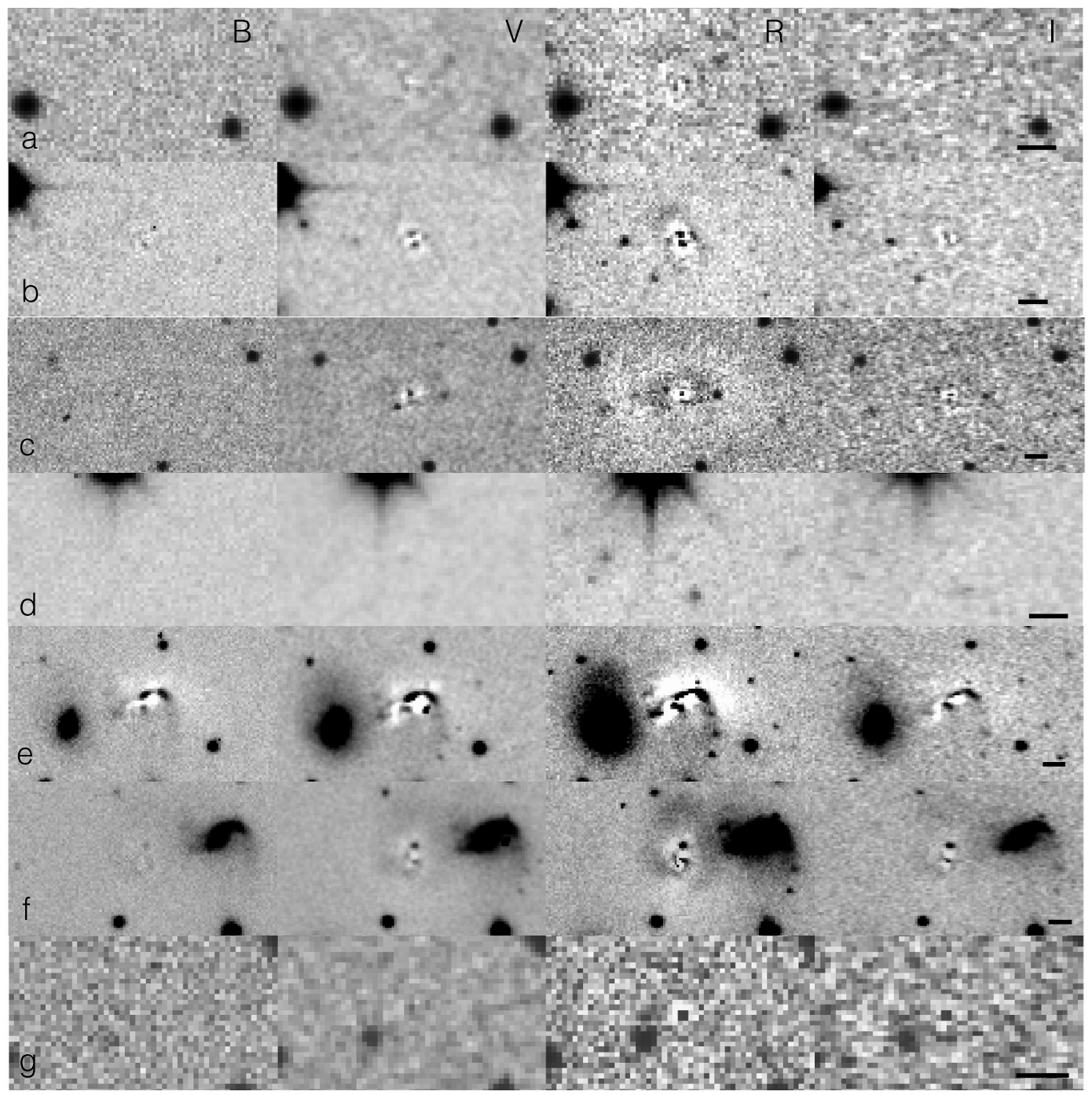

Fig. 3. Thumbnails showing the $B V R I$-band residuals of the seven galaxies identified in Fig. 1, after subtraction of the GIM2D bidimensional best-fit models. Bars are the same as in Fig. 2.

Table 4. Magnitudes and colors ${ }^{\mathrm{a}}$.

\begin{tabular}{llllllll}
\hline \hline Obj. Id. $^{\mathrm{b}}$ & $B$ & $V$ & $R$ & $I$ & $B-V$ & $V-R$ & $V-I$ \\
\hline $\mathrm{a}$ & 17.96 & 16.75 & 16.07 & 15.38 & 1.21 & 0.68 & 1.38 \\
& $\mathbf{1 6 . 2 5}$ & $\mathbf{1 5 . 4 7}$ & $\mathbf{1 4 . 9 9}$ & $\mathbf{1 4 . 5 9}$ & $\mathbf{0 . 7 8}$ & $\mathbf{0 . 4 7}$ & $\mathbf{0 . 8 9}$ \\
$\mathrm{b}$ & 17.10 & 15.95 & 15.24 & 14.55 & 1.15 & 0.71 & 1.40 \\
& $\mathbf{1 5 . 9 1}$ & $\mathbf{1 5 . 0 5}$ & $\mathbf{1 4 . 4 8}$ & $\mathbf{1 3 . 9 8}$ & $\mathbf{0 . 8 6}$ & $\mathbf{0 . 5 8}$ & $\mathbf{1 . 0 7}$ \\
$\mathrm{c}$ & 16.64 & 15.41 & 14.62 & 14.00 & 1.23 & 0.79 & 1.41 \\
& $\mathbf{1 5 . 1 4}$ & $\mathbf{1 4 . 2 8}$ & $\mathbf{1 3 . 6 7}$ & $\mathbf{1 3 . 2 9}$ & $\mathbf{0 . 8 6}$ & $\mathbf{0 . 6 1}$ & $\mathbf{0 . 9 9}$ \\
$\mathrm{d}$ & 18.12 & 16.90 & 16.51 & 15.70 & 1.23 & 0.39 & 1.20 \\
& $\mathbf{1 5 . 1 1}$ & $\mathbf{1 4 . 5 8}$ & $\mathbf{1 4 . 5 4}$ & $\mathbf{1 4 . 2 2}$ & $\mathbf{0 . 5 4}$ & $\mathbf{0 . 0 4}$ & $\mathbf{0 . 3 6}$ \\
$\mathrm{e}$ & 16.13 & 15.69 & 14.98 & 14.30 & 0.44 & 0.72 & 1.39 \\
& $\mathbf{1 4 . 2 2}$ & $\mathbf{1 4 . 2 3}$ & $\mathbf{1 3 . 7 4}$ & $\mathbf{1 3 . 3 6}$ & -0.02 & $\mathbf{0 . 5 0}$ & $\mathbf{0 . 8 7}$ \\
$\mathrm{f}$ & 16.85 & 15.47 & 14.84 & 14.20 & 1.38 & 0.63 & 1.27 \\
& $\mathbf{1 5 . 6 6}$ & $\mathbf{1 4 . 5 7}$ & $\mathbf{1 4 . 0 8}$ & $\mathbf{1 3 . 6 3}$ & $\mathbf{1 . 0 9}$ & $\mathbf{0 . 4 9}$ & $\mathbf{0 . 9 4}$ \\
$\mathrm{g}$ & 19.14 & 17.18 & 16.58 & 15.91 & 1.96 & 0.60 & 1.27 \\
& $\mathbf{1 7 . 9 5}$ & $\mathbf{1 6 . 2 8}$ & $\mathbf{1 5 . 8 2}$ & $\mathbf{1 5 . 3 4}$ & $\mathbf{1 . 6 7}$ & $\mathbf{0 . 4 6}$ & $\mathbf{0 . 9 4}$ \\
\hline
\end{tabular}

a Values in bold are corrected for inclination and total Galactic foreground extinction.

b Objects are labeled according to Fig. 1. 


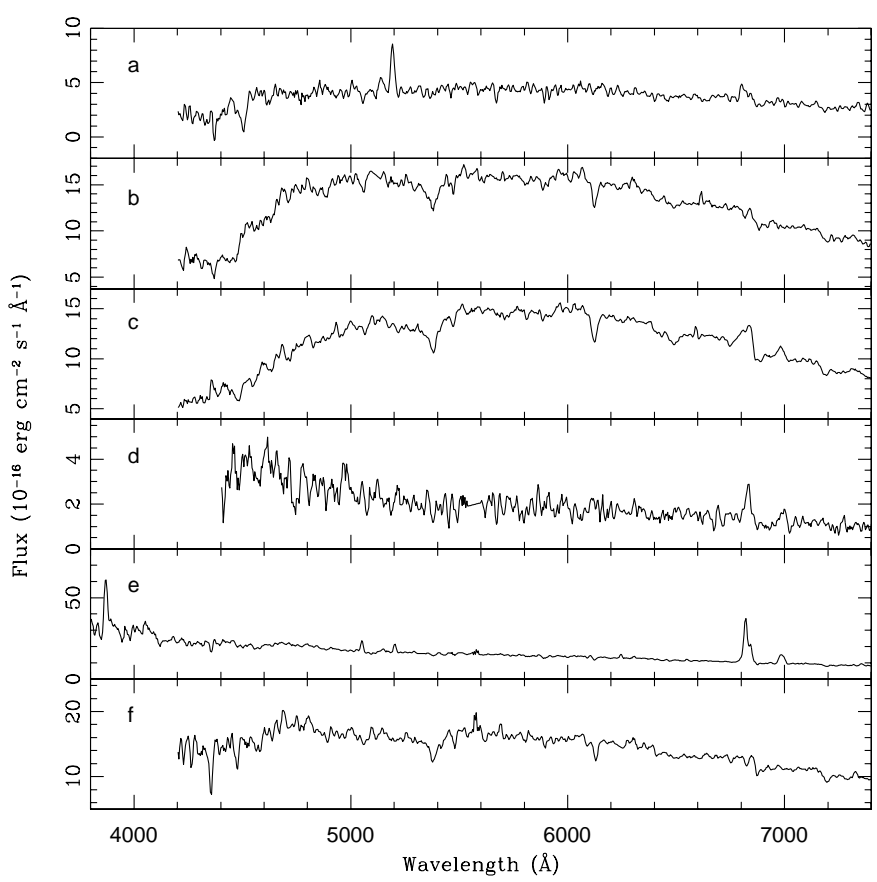

Fig. 4. Optical spectra of the six galaxies identified as members of the galaxy group CG J0247+44.9, labeled according to Fig. 1. Emission lines are visible in objects "a", "c", "d", and "e" with different properties (see text for a detailed description).

Table 5. Radial velocities.

\begin{tabular}{llll}
\hline \hline Obj. Id. & $\begin{array}{l}\alpha(\mathrm{J} 2000) \\
(\mathrm{h} \mathrm{m} \mathrm{s})\end{array}$ & $\begin{array}{l}\delta(\mathrm{J} 2000) \\
\left({ }^{\circ},{ }^{\prime \prime}\right)\end{array}$ & $\begin{array}{l}V_{\text {hel }} \\
\left(\mathrm{km} \mathrm{s}^{-1}\right)\end{array}$ \\
\hline CG J0247+449a & 024725.2 & 445039 & $10981 \pm 57$ \\
CG J0247+449b & 024737.6 & 445145 & $11346 \pm 116$ \\
CG J0247+449c & 024746.3 & 445258 & $11386 \pm 101$ \\
CG J0247+449d & 024749.8 & 445326 & $12240 \pm 32$ \\
CG J0247+449e & 024741.9 & 445034 & $11707 \pm 41$ \\
CG J0247+449f & 024740.4 & 445029 & $11931 \pm 88$ \\
2MASXi J0247387+445008 & 024738.7 & 445008 & $51000(?)$ \\
\hline
\end{tabular}

we observe an $\mathrm{H} \alpha / \mathrm{H} \beta$ ratio $\sim 2.89$, very close to the theoretical value of 2.86 for Case $\mathrm{B}$ recombination at electronic temperature $T_{\mathrm{e}}=10^{4} \mathrm{~K}$ (Osterbrock 1989), indicating very low internal extinction. The logarithmic ratios [O III] $5007 / \mathrm{H} \beta=0.95 \pm 0.34$ and $[\mathrm{N} \mathrm{II}] 6583 / \mathrm{H} \alpha=-0.30 \pm 0.18$, even with large uncertainties, are typical of a Seyfert-2 nucleus (Veilleux \& Osterbrock 1987).

$C G$ J0247+449b. This galaxy (2MASXi J0247376+ 445145 ) shows a $B / T$ ratio $\sim 0.57$, almost constant in all bands (see Table 3 ). The bulge is well reproduced by a de Vaucouleurs $r^{1 / 4}$-law, therefore we classify this object as E/S0. The residuals show in each band a ring-like structure at a radius of $\sim 4$ arcsec (better visible in the $R$-band in Fig. 3 ) and a central X-structure aligned with the major and minor photometric axes, which indicates a disky shape of the inner isophotes (Schweizer 1998). Numerical simulations (Naab \& Burkert 2001) have shown that faint ellipticals with disky isophotes might be the final product of unequal mass disk galaxy mergers (mass ratio $3: 1$ or 4:1).
The integrated spectrum of the galaxy shows only absorption lines and a continuum typical of an early-type galaxy (Fig. 4).

$C G \quad J 0247+449 c$. This galaxy (2MASXi J0247463+ 445258 ) has a $B / T$ ratio around or even less than $\sim 0.4$. Its bulge is well fitted by a de Vaucouleurs $r^{1 / 4}$-law like for object "b". So we classify it as S0/Sa. The residuals in VRI bands show the presence of a nuclear source, confirmed by the analysis of the optical spectrum. Indeed, the spectrum has the continuum of an early-type spiral (see Fig. 4) with deep metal absorption lines but with $\mathrm{H} \alpha$, [N II]6583 and [S II]6724 emissions. Since the [N II]6583 line appears more intense than $\mathrm{H} \alpha$ and the [O III]5007 is barely detectable at noise level, we suggest that this galaxy could host a LINER. In order to avoid an underestimate of the $\mathrm{H} \alpha$ flux due to the underlying stellar absorption, we applied a template correction, as in the case of object "a". After this correction the two emission lines $\mathrm{H} \alpha$ and [N II]6583 have comparable intensity. The diagnostic logarithmic emission-line ratios [N II] $6583 / \mathrm{H} \alpha$ and $[\mathrm{S} \mathrm{II}] 6724 / \mathrm{H} \alpha$ are $0.22 \pm 0.10$ and $-0.16 \pm 0.15$, respectively, confirming the classification as a LINER.

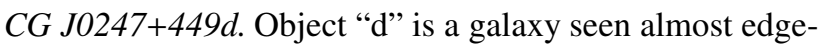
on $\left(i \sim 80^{\circ}\right)$. Although it is generally not recommended for highly inclined galaxies, we attempted a morphological analysis in this case, as well. Despite the lack of visible residuals after the subtraction of the bidimensional galaxy model in the four photometric bands (Fig. 3), the output values of the fit (Table 3) are somehow puzzling, suggesting that the bulge-disk decomposition mostly failed. Therefore we limit our comments to point out that the galaxy is significantly asymmetric and the bulge seems to follow an exponential law. We propose a Sbc classification for this object. The optical spectrum is very faint, but weak extended [S II]6724 and $\mathrm{H} \alpha$ emission lines are measurable and their logarithmic ratio is $-0.26 \pm 0.21$, somewhat higher than expected for H II-like galaxies. However the nondetection of [N II]6583 and [O III]5007 indicates a low ionization degree of the gas. The $\mathrm{H} \beta$ emission-line is detectable at noise level. We interpret this emission spectrum as an indication of a normal level of star formation for a late-type spiral galaxy.

$C G$ J0247+449e/f. These two galaxies form a close pair known as IRAS $02443+4437$. Despite the application of the maximum entropy algorithm to the far infrared (FIR) raw data extracted from the IRAS database to approach the diffraction limit of the telescope, the galaxy pair could not be resolved. A single infrared source, centered at the position of galaxy "f $\mathrm{f}$ ", is detectable only at 60 (Fig. 5) and $100 \mu \mathrm{m}$ with measured fluxes $0.618 \pm 0.005$ and $1.313 \pm 0.085$, respectively. The total FIR luminosity between 40 and $120 \mu \mathrm{m}$ calculated following Helou et al. (1985) is $L_{\mathrm{FIR}}=2.75 \times 10^{10} L_{\odot}$, which yields a total star formation rate $S F R=14.4 M_{\odot}$ (Hunter et al. 1986). The spectroscopic analysis reveals that the FIR emission is dominated by galaxy "e", which shows bright emission lines typical of a starburst galaxy. Measured fluxes of the emission lines (Table 6) have been corrected for internal extinction $A_{\mathrm{V}}=1.61$ determined assuming a theoretical $\mathrm{H} \alpha / \mathrm{H} \beta=2.86$ and applying the CCM extinction law. The logarithmic diagnostic ratios $[\mathrm{N} \mathrm{II}] 6583 / \mathrm{H} \alpha=-0.41 \pm 0.02$, 
[S II] $6724 / \mathrm{H} \alpha=-0.51 \pm 0.04,[\mathrm{O} \mathrm{I}] 6300 / \mathrm{H} \alpha=-1.61 \pm 0.13$, and $[\mathrm{O} \mathrm{III}] 5007 / \mathrm{H} \beta=-0.16 \pm 0.06$ confirm the thermal nature of the ionizing source. The reddening corrected $\mathrm{H} \alpha$ luminosity $L(\mathrm{H} \alpha)=5.1 \times 10^{41} \mathrm{erg} \mathrm{s}^{-1}$ corresponds to a $S F R \simeq 4 M_{\odot} \mathrm{yr}^{-1}$ (Kennicutt 1998). This value is lower than the SFR of the galaxy pair estimated from the FIR luminosity, but it only accounts for the portion of the galaxy covered by the slit. The spectrum of object "f $\mathrm{f}$ " shows the typical absorption features of an early-type galaxy, without any emission line, but with a relatively blue continuum. The morphological analysis confirms the spectroscopic results. Galaxy "e" is clearly a spiral with irregular shape, as indicated by the high value of the asymmetry index, which made less accurate the bidimensional fit. In fact strong residuals remain where star forming regions are located. These regions, brighter in the nucleus and fainter in the inner spiral arms, are likely to be responsible for the relatively high value of the obtained $B / T$ ratio $(\sim 0.37)$, and for the negative $B-V$ color (see Table 4). Since the Sérsic index of the bulge results $<1$, we suggest a Sbc classification for this object. Galaxy "f" shows a mean $B / T$ ratio of $\sim 0.7$ and its bulge is well fitted by a de Vaucouleurs $r^{1 / 4}$-law, indicative of a morphological type $\mathrm{E} / \mathrm{S} 0$. A moderate asymmetry is present (Table 3) and the residuals show in all bands an arm-like structure, which departs from the south of the nucleus winding around it, and a blob north of the nucleus. The morphological distortions observed in these galaxies together with their accordant redshifts indicate that this is a real interacting pair. As demonstrated by some authors (Rampazzo \& Sulentic 1992; Hernández Toledo et al. 1999) galaxy pairs with mixed morphology exist in significant numbers and are believed to be the product of interaction phenomena. In agreement with our results about this galaxy pair, it is found that spiral + lenticular systems show an enhancement in the FIR emission with increased rate and efficiency of induced star formation (Hernández Toledo et al. 2001).

\section{Discussion}

As shown in Sect. 3 on the basis of radial velocity measurements, 6 out of the 7 galaxies grouped around the barycentric position $\alpha(\mathrm{J} 2000)=02^{\mathrm{h}} 47^{\mathrm{m}} 36^{\mathrm{s}} .3, \delta(\mathrm{J} 2000)=44^{\circ} 51^{\prime} 39^{\prime \prime}$, form a physical system with a median radial velocity $V=$ $11730 \mathrm{~km} \mathrm{~s}^{-1}$. The group, whose ellipticity calculated following Rood (1979) is $\epsilon=0.68$, shows an elongated shape, a property that have been found to be typical of galaxy groups and interpreted as indication of three-dimensional shapes intrinsically prolate (Hickson 1997, and references therein).

Although the group does not exhibit a particularly compact configuration, we find that its properties fulfill all criteria applied by Hickson (Hickson 1982) to define compact groups of galaxies (CGs). In fact the median projected separation of our group, $R=121.7 \mathrm{kpc}^{4}$, is significantly higher than the values observed in the most compact systems, like Seyfert's Sextet or HCG 31 (Hickson 1997), but still within the range of $R$ measured in the Hickson's sample (HCGs). The average surface

\footnotetext{
${ }^{4}$ Throughout this paper we assume $H_{0}=75 \mathrm{~km} \mathrm{~s}^{-1}$, unless otherwise specified.
}

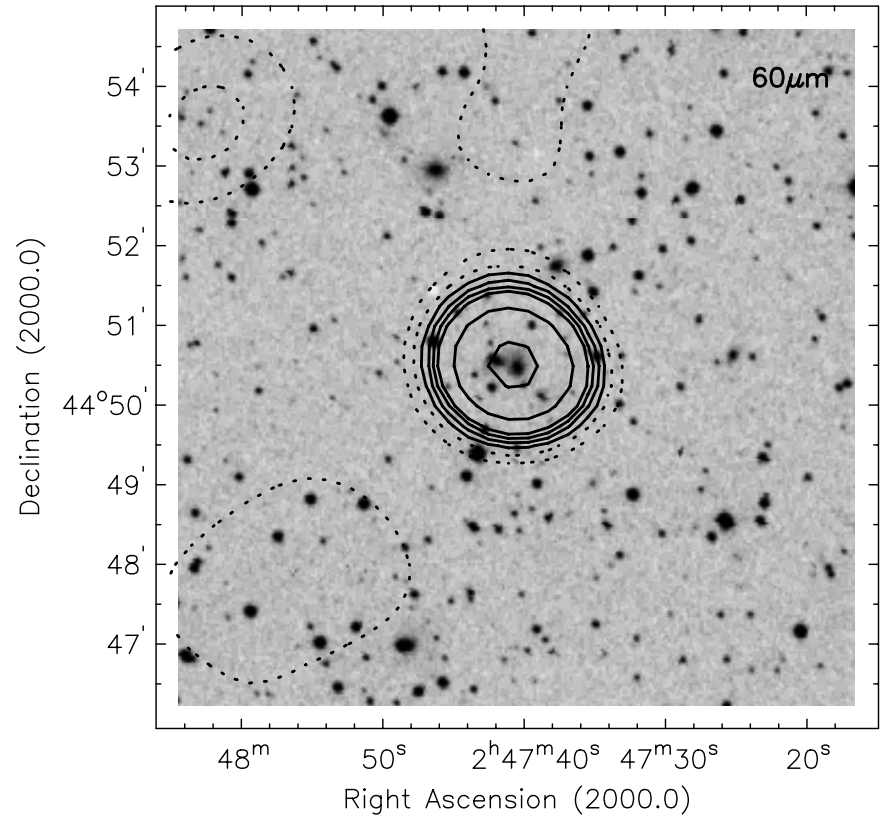

Fig. 5. Contour map of the $60 \mu \mathrm{m}$ IRAS emission of the galaxy pair IRAS $02443+4437$, after application of the maximum entropy algorithm, overplotted to the Digitized Sky Survey optical image. Dashed contours are drawn at $1 \sigma$ and $2 \sigma$ level over the background; solid contours are drawn at $3,4,5,6,10$, and $20 \sigma$ levels.

Table 6. Emission line fluxes of CG J0247+449e.

\begin{tabular}{lll}
\hline \hline Line & $F_{\lambda} / F_{\beta}$ & $I_{\lambda} / I_{\beta}$ \\
\hline$[\mathrm{O} \mathrm{II}] \lambda 3727$ & $5.56 \pm 1.18$ & 9.70 \\
{$[\mathrm{O} \mathrm{III}] \lambda 4959$} & $0.30 \pm 0.08$ & 0.29 \\
{$[\mathrm{O} \mathrm{III}] \lambda 5007$} & $0.75 \pm 0.11$ & 0.70 \\
$\mathrm{He}$ I $\lambda 5876$ & $0.21 \pm 0.06$ & 0.15 \\
{$[\mathrm{O} \mathrm{I}] \lambda 6300$} & $0.11 \pm 0.04$ & 0.07 \\
{$[\mathrm{~N} \mathrm{II}] \lambda 6548$} & $0.50 \pm 0.09$ & 0.30 \\
$\mathrm{H} \alpha$ & $4.77 \pm 0.36$ & 2.86 \\
{$[\mathrm{~N} \mathrm{II}] \lambda 6583$} & $1.86 \pm 0.18$ & 1.11 \\
{$[\mathrm{~S} \mathrm{II}] \lambda 6716$} & $0.84 \pm 0.12$ & 0.48 \\
{$[\mathrm{~S} \mathrm{II}] \lambda 6731$} & $0.71 \pm 0.11$ & 0.41 \\
& & \\
$E(B-V)=0.52 \pm 0.08$ & & \\
$I_{\beta}=6.04 \times 10^{-14}$ & $\left(\mathrm{erg} \mathrm{cm}^{-2} \mathrm{~s}^{-1}\right)$ & \\
\hline
\end{tabular}

brightness in $R$ band, $\mu_{R}=24.4$ mag $\operatorname{arcsec}^{-2}$, evaluated within the minimum circle containing the centers of all galaxy members, having radius $\theta_{R} \sim 2^{\prime} .6$, satisfies the compactness criterion, requiring $\mu_{R}<26.0 \mathrm{mag} \operatorname{arcsec}^{-2}$. The isolation of the group is assured by the fact that the nearest cataloged galaxy of comparable magnitude (2MASXi J0246441+444820) is at a distance $3.7 \theta_{R}$ from the group's center, therefore well outside the isolation radius $\theta_{N}=3 \theta_{R}$.

Moreover, as we have shown in Sect. 4, CG J0247+44.9 exhibits a wide range of activity types, being characterized by the presence of a Seyfert 2 galaxy, a LINER, a HII galaxy and a strongly interacting starburst galaxy. The two active nuclei are hosted by early-type spirals. This is in agreement with Coziol et al. (1998a,b), who found in a sample of 17 HCGs 
that the AGNs are preferentially located in the most early-type galaxies and that $50 \%$ of them are low-luminosity ones, like Seyfert 2 or LINER. They also pointed out that AGNs are systematically concentrated toward the central parts of the groups, while starburst galaxies are distributed in the external parts. This is not verified in our case, as the two active galaxies of CG J0247+44.9 are located in the outer parts of the group, while the starburst galaxy is near the geometrical central position. It is interesting to note that the closest object to the geometrical center of the group is the non-active, early-type galaxy "b", whose internal structures (see Sect. 4) might be the outcome of an unequal mass, disk galaxy merger.

A good agreement has also been found between other properties of CG J0247+449 and those of HCGs. In particular the velocity dispersion $\sigma_{\mathrm{V}}=458 \mathrm{~km} \mathrm{~s}^{-1}$ is lower than the maximum observed value for HCGs, albeit higher than the median of $200 \mathrm{~km} \mathrm{~s}^{-1}$ (Hickson et al. 1992). The intrinsic three-dimensional velocity dispersion $\sigma_{3 \mathrm{D}}=709.5 \mathrm{~km} \mathrm{~s}^{-1}$ calculated taking into account the velocity errors, has been used to estimate the dimensionless crossing time $H_{0} t_{\mathrm{c}}$, which gives an indication of the dynamical state of the group. We have found a value of 0.016 that, when compared with the fraction of late-type spirals in the group $\left(f_{\mathrm{s}}=0.34\right)$, results in good agreement with the $\log H_{0} t_{\mathrm{c}}-f_{\mathrm{s}}$ relationship found by Hickson et al. (1992). According to this relationship, the spiral fraction is lower on average in groups with small crossing times, as expected in case of hierarchical evolution within galaxy groups. Since numerical simulations (Perea et al. 1990) have shown that a group should approach the virial equilibrium condition after about three crossing times and our group has a relatively low $t_{\mathrm{c}}$, we calculated the virial mass following Perea et al. (1990) and found a value of $\sim 1.5 \times 10^{13} M_{\odot}$, comparable with the virial masses of HCGs, which reach a maximum value of $7.3 \times 10^{13} M_{\odot}$. It must be stressed that the assumption of virial equilibrium and the neglect of the mass spectrum of the galaxies could lead to a large overestimate of the virial mass. Assuming as $B$ luminosity of the group the luminosity of all the accordant galaxies, $L_{B}=1.95 \times 10^{11} L_{\odot}$, we found a mass-tolight ratio $M / L_{B} \sim 77$, somewhat higher than the median value for HCGs $\left(M / L_{B}=37.5\right)$, but significantly smaller than typical values for loose groups (Hickson et al. 1992, and references therein).

On the basis of the above observational properties we suggest that CG J0247+44.9 is a dynamically old but still evolving compact galaxy group, as indicated by the strong signs of interaction in the close pair IRAS $02443+4437$ belonging to the system. This idea is in agreement with the relatively high velocity dispersion of the group, typical of groups having a high fraction of early type galaxies (Hickson 1997). These systems often show an extended soft X-ray halo (Mulchaey 2000 , and references therein) suggesting that they are physically bound and - at least in case of symmetric X-ray morphologies - already relaxed, therefore dynamically old groups. The ROSAT All-Sky Survey (RASS) shows no obvious excess of X-ray emission at the location of CG J0247+44.9. To check whether this is consistent with the presence of a galaxy group, we have estimated the expected soft X-ray luminosity and temperature of our group by means of the empirical $L_{\mathrm{X}}-\sigma_{V}$ and
$T-\sigma_{V}$ relations from Mulchaey \& Zabludoff (1998) obtaining $L_{\mathrm{X}}=5.96 \times 10^{42} \mathrm{erg} \mathrm{s}^{-1}$ and $T=1.38 \mathrm{keV}$. At the distance of the source $(D \sim 156.4 \mathrm{Mpc})$ this luminosity corresponds to a flux of $\sim 2.03 \times 10^{-12} \mathrm{erg} \mathrm{cm}^{-2} \mathrm{~s}^{-1}$, a value approaching the $3 \sigma$ upper limit to the X-ray flux derived from RASS data within a $250 \mathrm{~h}^{-1} \mathrm{kpc}\left(h=H_{0} / 100\right)$ aperture after correction for the Galactic neutral hydrogen column density in the direction of the group (H. Ebeling, private communication). Therefore the lack of cataloged RASS X-ray sources at the location of CG J0247+44.9 is not inconsistent with our results.

\section{Conclusions}

We have identified a new compact group of galaxies at low Galactic latitude and analyzed the main spectroscopic and morphological features of its members. The general properties of the system have been found in good agreement with those of Hickson Compact Groups. In summary this group, named as CG J0247+44.9, is composed by six members, including the close interacting S0+S pair, IRAS $02443+4437$, whose enhanced infrared emission is dominated by the spiral component. Four out of the six galaxies show some kind of activity, ranging from a moderate level of star formation to a Seyfert nucleus. The closest galaxy to the geometrical center of the group is an $\mathrm{E} / \mathrm{S} 0$ with internal structures suggestive of a past history of interaction or even merger. The early-type morphology $(\mathrm{S} 0 / \mathrm{Sa})$ is dominant in the group, which comprises only two late-type spirals. In the framework of hierarchical evolution, where spirals are transformed into ellipticals through interactions and mergers, this property combined with the short crossing time and the relatively high velocity dispersion suggests that the group is dynamically old. However the evident strong signs of interactions in the close pair indicate that the system is still evolving. High spatial and spectral resolution kinematics would be necessary to better establish the dynamical state of this galaxy group and the interaction history of its members.

Acknowledgements. We are grateful to Harald Ebeling for having provided us with the analysis of ROSAT data and for the useful discussion, and to Ronald Weinberger for a careful reading of the manuscript and useful suggestions. SC is grateful to the Institut für Astrophysik Innsbruck, for warm hospitality and to the Astrophysikalisches Institut Potsdam for the access to the IRAS data processing with the maximum entropy program. ST acknowledges support by the Austrian Science Fund (FWF) under project no. P15065. We wish also to thank the technicians of Asiago Observatory for their assistance during the observations.

\section{References}

Andredakis, Y. C. 1998, MNRAS, 295, 725

Andredakis, Y. C., \& Sanders, R. H. 1994, MNRAS, 267, 283

Bertin, E., \& Arnouts, S. 1996, A\&A, 117, 393

Burstein, D., \& Heiles, C. 1982, AJ, 87, 1165

Buta, R., \& Williams, K. L. 1995, AJ, 109, 543

Cardelli, J. A., Clayton, G. C., \& Mathis, J. S. 1989, ApJ, 345, 245 (CCM)

Carollo, C. M., Stiavelli, M., de Zeeuw, P. T., Seigar, M., \& Dejonghe, H. 2001, ApJ, 546, 216 
Coziol, R., Ribeiro, A. L. B., de Carvalho, R. R., \& Capelato, H. V. 1998a, ApJ, 493, 563

Coziol, R., de Carvalho, R. R., Capelato, H. V., \& Ribeiro, A. L. B. 1998b, ApJ, 506, 545

de Vaucouleurs, G. 1948, Ann. Astrophys., 11, 247

de Vaucouleurs, G., de Vaucouleurs, A., Corwin, H., et al. 1991, Third Reference Catalogue of Bright Galaxies (Springer, New York)

Helou, G., Soifer, B. T., \& Rowan-Robinson, M. 1985, ApJ, 298, L7

Hernández Toledo, H. M., Dultzin-Hacyan, D., Gonzalez, J. J., \& Sulentic, J. W. 1999, AJ, 118, 108

Hernández Toledo, H. M., Dultzin-Hacyan, D., \& Sulentic, J. W. 2001, AJ, 121, 1319

Hickson, P. 1982, ApJ, 255, 382

Hickson, P. 1997, ARA\&A, 35, 357

Hickson, P., Mendes de Oliveira, C., Huchra, J. P., \& Palumbo, G. G. C. 1992, ApJ, 399, 353

Ho, L. C., Filippenko, A. V., \& Sargent, W. L. W. 1993, ApJ, 417, 63

Huchra, P. J., Mader, J., Schneider, S. E., et al. 2000, ASP Conf. Ser., 218, 101

Hunter, D. A., Gillet, F. C., Gallagher, J. S., Rice, W. L., \& Low, F. J. 1986, ApJ, 303, 171

Kennicutt, R. C. Jr. 1998, ApJ, 498, 541

Kraan-Korteweg, R. C., \& Lahav, O. 2000, A\&A Rev., 10, 211

Kinney, A. L., Calzetti, D., Bohlin, R., et al. 1996, ApJ, 467, 38

Landolt, A. U. 1992, AJ, 104, 340

Liu, C. T. 1999, ASP Conf. Ser., 191, 99

Mulchaey, J. S. 2000, ARA\&A, 38, 289

Mulchaey, J. S., \& Zabludoff, A. I. 1998, ApJ, 496, 73
Naab, T., \& Burkert, A. 2001, preprint [astro-ph/0110179]

Nakanishi, K., Takata, T., Yamada, T., et al. 1997, ApJS, 112, 245

Osterbrock, D. E. 1989, Astrophysics of Gaseous Nebulae and Active Galactic Nuclei (Mill Valley, CA: Univ. Science Books)

Perea, J., Del Olmo, A., \& Moles, M. 1990, A\&A, 237, 319

Rampazzo, R., \& Sulentic, J. W. 1992, A\&A, 259, 43

Rood, H. J. 1979, ApJ, 233, 21

Schlegel, D. J., Finkbeiner, D. P., \& Davis, M. 1998, ApJ, 500, 525

Schweizer, F. 1998, in Galaxies: Interactions and Induced Star Formation, Saas-Fee Advanced Course 26, Lecture Notes 1996 (Springer-Verlag Berlin/Heidelberg; ISBN: 3-540-63569-6)

Sérsic, J. L. 1968, Atlas de galaxias australes, Observatorio Astronomico, Cordoba

Simard, L. 1998, in Astronomical Data Analysis Software Systems VII, ed. R. Albrecht, R. N. Hook, \& H. A. Bushouse, ASP Conf. Ser., 145,108

Simard, L., et al. 2001, preprint, http://www.hia.nrc.ca/STAFF/lsd/gim2d/paper/

Simien, F., \& de Vaucouleurs, G. 1986, ApJ, 302, 564

Stetson, P. B., \& Harris, W. E. 1988, AJ, 96, 909

Tonry, J. L., \& Davis, M. 1979, AJ, 84, 1511

Tran, K.-V. H., Simard, L., Zabludoff, A. I., \& Mulchaey, J. S. 2001, ApJ, 549, 172

Veilleux, S., \& Osterbrock, D. E. 1987, ApJS, 63, 295

Weinberger, R., Saurer, W., Marchiotto, W., et al. 2000, in Mapping the Hidden Universe: The Universe Behind the Milky Way - The Universe in H I, ed. R. C. Kraan-Korteweg, P. A. Henning, \& H. Andernach, ASP Conf. Ser., 218, 27 